\title{
NON-INVASIVE ISTHMUS IDENTIFICATION OF COMPLEX ARRHYTHMIAS IN CONGENITAL HEART DISEASE
}

Levio Quinto ${ }^{1}$, Paula Sanchez ${ }^{1}$, Francisco Alarcón ${ }^{1}$, Silvia Montserrat ${ }^{2}$, Susanna

Prat-Gonzalez ${ }^{2}$, Eduard Guasch ${ }^{1}$, Josep Brugada ${ }^{1}$, Jose Maria Tolosana ${ }^{1}$, Luis Mont Girbau $^{1}$, and Ivo Roca-Luque ${ }^{1}$

${ }^{1}$ Hospital Clinic de Barcelona

${ }^{2}$ Cardiovascular Imaging Unit

June 15, 2020

\section{Hosted file}

NON-INVASIVE ISTHMUS IDENTIFICATION OF COMPLEX ARRHYTHMIAS IN CONGENITAL HEART DISEASE.dOCX available at https://authorea.com/users/333771/articles/459879-non-invasive-isthmusidentification-of-complex-arrhythmias-in-congenital-heart-disease 